\title{
A case of appendix adenocarcinoma associated with ulcerative colitis.
}

\author{
Yohsuke Fukumoto $^{1}$, Yuh Kobayashi ${ }^{1}$, Shizuki Takemura ${ }^{1}$, Kiyosumi Maeda ${ }^{1}$, Fumiyasu \\ Nakamura $^{1}$, Osamu Inatomi ${ }^{2}$, Akira Andoh ${ }^{2}$, and Hiromitsu Ban ${ }^{1}$ \\ ${ }^{1}$ Kusatsu General Hospital \\ ${ }^{2}$ Shiga University of Medical Science
}

July 27, 2021

\begin{abstract}
Appendix adenocarcinoma associated with ulcerative colitis (UC) is extremely rare. Pathological examination of a man with 6year history of UC revealed a primary appendix adenocarcinoma mixed with adenoma component. Dysplasia was not identified.

The adenoma-carcinoma sequence, but not the dysplasia-carcinoma sequence, might be involved in this UC-associated appendix adenocarcinoma.
\end{abstract}

\section{Introduction}

Ulcerative colitis (UC) is a chronic relapsing inflammatory disorder of the colon (13). Patients with UC have an increased risk for developing colorectal cancer (CRC), known as UC-associated CRC $(3,8,18)$. The cumulative incidence of $\mathrm{UC}$-associated $\mathrm{CRC}$ has been reported to be from $7.5 \%$ to $18.4 \%$ at 30 years after onset (20). However, appendix adenocarcinoma associated with UC is extremely rare.

\section{Case report}

A 52-year-old man with a 6-year history of UC (left-sided colitis) was admitted to our hospital because of umbilical pain and low-grade fever. Oral administration of 5-aminosalicylic acid had been discontinued one year earlier due to clinical remission. A colonoscopy performed one year earlier showed an endoscopic remission. At this time, a mild inflammation was observed around the appendix orifice. He had no family history of malignancies.

Physical examination on admission showed tenderness in the right lower quadrant of the abdomen, but there was no peritoneal irritation sign. Laboratory examination revealed an elevation of C-reactive protein $(2.01 \mathrm{mg} / \mathrm{dl})$, but tumor markers such as carcinoembryonic antigen and carbohydrate antigen 19-9 were within normal range. A contrast material-enhanced computed tomography scan showed a stenotic change of the proximal ascending colon by a mass showing extramural growth (Figure1A). The extramural part of the mass extended to the dilated appendix with intraluminal tumor growth (Figure1B). A colonoscopy on admission showed a circumferential stenosis of the proximal ascending colon and the lumen was spread by air supply, suggesting extramural compression. (Figure 2). The mucosa surface was reddish but intraluminal growth of the tumor was not observed. Pathological examination of biopsy specimens showed no malignancy.

A laparoscope-assisted right hemicolectomy was performed on the $9^{\text {th }}$ day after admission. Macroscopically, the appendix was dilated and strongly adhered to the proximal portion of the ascending colon (Stage IIIB [T4b, N1a, M0] according to the TNM classification). Pathological examination revealed a primary adenocarcinoma mixed with an adenoma component of the appendix (Figure 3). The tumor invaded the serosal 
side of the proximal ascending colon. Dysplasia was not identified in any pathologically examined specimens. Genomic analysis of carcinoma cells revealed the KRAS G13D mutation. His postoperative course was uneventful, and adjuvant chemotherapy was started from 6 weeks after surgery.

\section{Discussion}

Appendix carcinoma is rare, and accounts for $0.4 \%$ of all gastrointestinal malignancies(15). Furthermore, UCassociated appendix carcinoma is extremely rare, and only a limited number of cases have been reported (11, $16,17,19)$. The homology of pathogenesis between UC-associated appendix carcinoma and UC-associated CRC remains poorly understood.

Chronic mucosal inflammation is considered to be a main driver of UC-associated CRC $(6,7)$, and established risk factors include prolonged disease duration, extensive colonic involvement, family history of CRC, and coexistence of primary sclerosing cholangitis $(2,4,6,7)$. The severity of histologic inflammation is an independent risk factor (7). However, the relationship between long-term mucosal inflammation and appendix carcinoma remains unclear, because previously published cases have shown that appendix carcinoma developed not only in patients with pancolitis but also in those with left-sided colitis.

Sporadic CRC develops through the adenoma-carcinoma sequence (4), but UC-associated CRC is characterized by the inflammation-dysplasia-carcinoma sequence (5). A typical feature of UC is a continuous mucosal inflammatory lesion extending proximally from the rectum without any skip lesion. However, previous studies have reported that isolated peri-appendiceal inflammation is frequently observed as a "skip lesion" in more than half of patients with left-sided colitis or distal type colitis $(9,12)$. In this case, a colonoscopy performed one year earlier had revealed an isolated inflammation (mucosal redness and granularity) surrounding the appendix orifice. These findings indicate the presence of sustained inflammation in the appendix of patients with left-sided colitis as well as those with pancolitis, raising the possibility that like UC-associated CRC, continuous inflammation may be associated with the development of appendix carcinoma.

On the other hand, there are contrasting reports on the contribution of the inflammation-dysplasia sequence to UC-associated appendix adenocarcinoma. The presence of dysplasia is an important finding for the diagnosis of UC-associated CRC. However, dysplasia of the colonic mucosa was not identified in some previously published cases of UC-associated appendix carcinoma nor in the current case $(11,16,19)$. Furthermore, pathological examination in this case showed the presence of an adenoma component. These suggest that some UC-associated appendix carcinomas might develop via the non-inflammatory carcinogenesis pathway.

Advances in the genetic approaches to cancer have led to the characterization of the genomic landscape of various types of tumors. Robles et al. revealed that the KRAS mutation was detected at a significantly lower rate in UC-associated CRC than in sporadic CRC (14). Matsumoto et al . recently reported that UCassociated CRC developed through the inflammatory carcinogenesis pathway is characterized by the TP53 mutation but those through the noninflammatory pathway are characterized by the KRAS mutation (10). We detected the KRAS G13D mutation. This finding also lends support to the theory of a contribution by the non-inflammatory carcinogenesis pathway in this case.

A preoperative diagnosis of appendix carcinoma has been reported to be extremely difficult, since there are no pathognomonic signs or symptoms (1). In this case, a colonoscopy performed one year earlier revealed no evidence of appendix carcinoma. Since more than $70 \%$ of patients with appendix carcinoma present with clinical symptoms of acute appendicitis (1), we should pay attention to such symptoms in the follow-up of $\mathrm{UC}$ patients presenting a skip lesion at the appendix orifice.

Finally, this case suggests an association between UC-associated appendix carcinoma and the noninflammatory carcinogenesis pathway. Genetic approaches will be helpful in defining the pathological mechanisms underlying this malignant disorder.

\section{Conflict interest}

None declared. 


\section{Author contribution}

All authors: contributed to the design of this manuscript. YK, AA and HB: wrote the first draft, ST: made a pathological diagnosis, KM: made CT diagnosis, FN and OI: scientifically reviewed the manuscript, AA and $\mathrm{BH}$ : wrote the final version.

\section{Reference}

1. Al-Wiswasy MKM, Al-Balas H, Al-Saffar RAS, and Al-Balas M. Synchronous primary adenocarcinoma of the appendix and colon: Case report and literature review. Int J Surg Case Rep 77: 628-633, 2020.

2. Colman RJ, and Rubin DT. Histological inflammation increases the risk of colorectal neoplasia in ulcerative colitis: a systematic review.Intest Res 14: 202-210, 2016.

3. Eaden JA, Abrams KR, and Mayberry JF. The risk of colorectal cancer in ulcerative colitis: a metaanalysis. Gut 48: 526-535, 2001.

4. Ekbom A, Helmick C, Zack M, and Adami HO. Ulcerative colitis and colorectal cancer. A populationbased study. N Engl J Med 323: 1228-1233, 1990.

5. Fearon ER. Molecular genetic studies of the adenoma-carcinoma sequence. Adv Intern Med 39: 123-147, 1994.

6. Flores BM, O'Connor A, and Moss AC. Impact of mucosal inflammation on risk of colorectal neoplasia in patients with ulcerative colitis: a systematic review and meta-analysis. Gastrointest Endosc 86: 1006-1011 e1008, 2017.

7. Gupta RB, Harpaz N, Itzkowitz S, Hossain S, Matula S, Kornbluth A, Bodian C, and Ullman T. Histologic inflammation is a risk factor for progression to colorectal neoplasia in ulcerative colitis: a cohort study. Gastroenterology 133: 1099-1105; quiz 1340-1091, 2007.

8. Herrinton LJ, Liu L, Levin TR, Allison JE, Lewis JD, and Velayos F. Incidence and mortality of colorectal adenocarcinoma in persons with inflammatory bowel disease from 1998 to 2010. Gastroenterology143: 382$389,2012$.

9. Ladefoged K, Munck LK, Jorgensen F, and Engel P. Skip inflammation of the appendiceal orifice: a prospective endoscopic study. Scand J Gastroenterol 40: 1192-1196, 2005.

10. Matsumoto K, Urabe Y, Oka S, Inagaki K, Tanaka H, Yuge R, Hayashi R, Kitadai Y, Arihiro K, Shimamoto F, Tanaka S, and Chayama K. Genomic Landscape of Early-stage Colorectal Neoplasia Developing From the Ulcerative Colitis Mucosa in the Japanese Population. Inflamm Bowel Dis 27: 686-696, 2021.

11. Nehme F, Schneider A, and Hamid F. Appendiceal Adenocarcinoma Associated With Ulcerative Colitis. ACG Case Rep J 6: e00255, 2019.

12. Park SH, Loftus EV, Jr., and Yang SK. Appendiceal skip inflammation and ulcerative colitis. Dig Dis Sci 59: 2050-2057, 2014.

13. Podolsky DK. Inflammatory bowel disease. The New England journal of medicine 347: 417-429, 2002.

14. Robles AI, Traverso G, Zhang M, Roberts NJ, Khan MA, Joseph C, Lauwers GY, Selaru FM, Popoli M, Pittman ME, Ke X, Hruban RH, Meltzer SJ, Kinzler KW, Vogelstein B, Harris CC, and Papadopoulos N. Whole-Exome Sequencing Analyses of Inflammatory Bowel Disease-Associated Colorectal Cancers. Gastroenterology 150: 931-943, 2016.

15. Ruoff C, Hanna L, Zhi W, Shahzad G, Gotlieb V, and Saif MW. Cancers of the appendix: review of the literatures. ISRN Oncol 2011: 728579, 2011.

16. Shen H, Lipka S, and Katz S. Appendiceal adenocarcinoma in a patient with chronic ulcerative colitis: what is the appropriate surgical procedure? J Crohns Colitis 8: 334-335, 2014. 
17. Takeda Y, Nakase H, Mikami S, Inoue T, Satou S, Sakai Y, and Chiba T. Possible link between ulcerative colitis and in situ adenocarcinoma of an appendiceal mucocele: importance of inflammation in the appendiceal orifice related to UC. Inflamm Bowel Dis 14: 873-874, 2008.

18. Verdon C, Aruljothy A, Lakatos PL, and Bessissow T. Endoscopic surveillance strategies for dysplasia in ulcerative colitis.Frontline Gastroenterol 11: 124-132, 2020.

19. Villanueva Saenz E, Perez-Aguirre J, Belmonte MC, Martinez PH, Marquez RM, and Carranza RJ. Appendix adenocarcinoma associated with ulcerative colitis: a case report and literature review. Tech Coloproctol 10: 54-56, 2006.

20. Watanabe T, Ajioka Y, Mitsuyama K, Watanabe K, Hanai H, Nakase H, Kunisaki R, Matsuda K, Iwakiri R, Hida N, Tanaka S, Takeuchi Y, Ohtsuka K, Murakami K, Kobayashi K, Iwao Y, Nagahori M, Iizuka B, Hata K, Igarashi M, Hirata I, Kudo SE, Matsumoto T, Ueno F, Watanabe G, Ikegami M, Ito Y, Oba K, Inoue E, Tomotsugu N, Takebayashi T, Sugihara K, Suzuki Y, Watanabe M, and Hibi T. Comparison of Targeted vs Random Biopsies for Surveillance of Ulcerative Colitis-Associated Colorectal Cancer. Gastroenterology 151: 1122-1130, 2016.

\section{Figure legends}

Figure 1. Computed tomography scan on admission. The appendix was dilated with the tumor exhibiting intraluminal growth. This tumor extended to the serosal side of the proximal ascending colon.

Figure 2. Endoscopic finding of the stenotic portion. Colonoscopy on admission showed a circumferential stenosis of the ascending colon. The lumen was spread by air supply, and intraluminal tumor growth was not observed. The mucosa was reddish but pathological examination of biopsy specimens showed no malignancy.

Figure 3. Pathological finding of the appendix. The tumor was arisen from the appendix wall and exhibited intraluminal growth. The tumor consisted mainly of carcinoma, but an adenoma component was also found. (A) Loupe image, (B) adenocarcinoma (x 200), and (C) adenoma component (x200).

Figure 1.
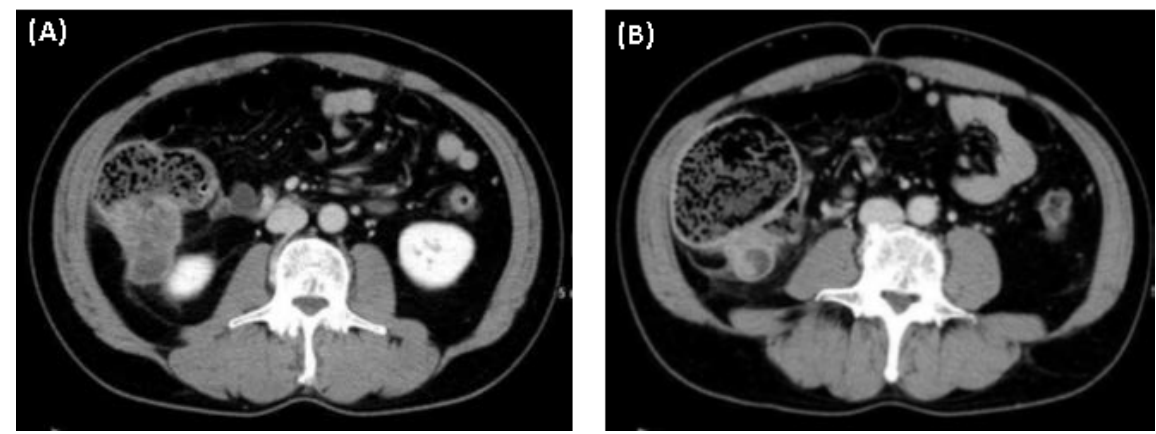
Figure 2.

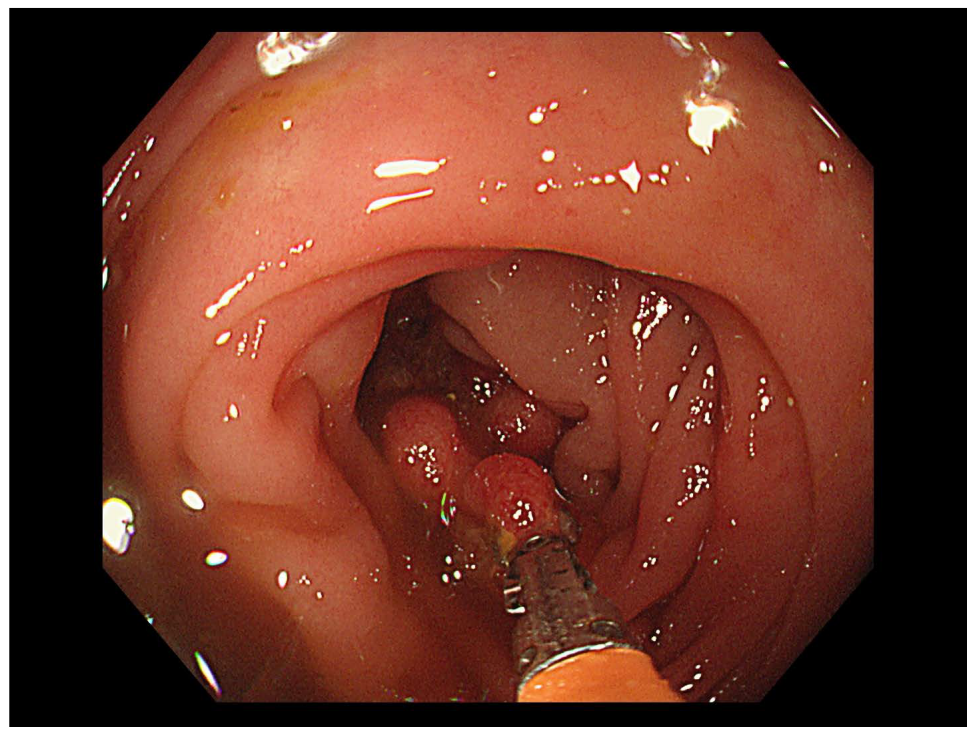

Figure 3.
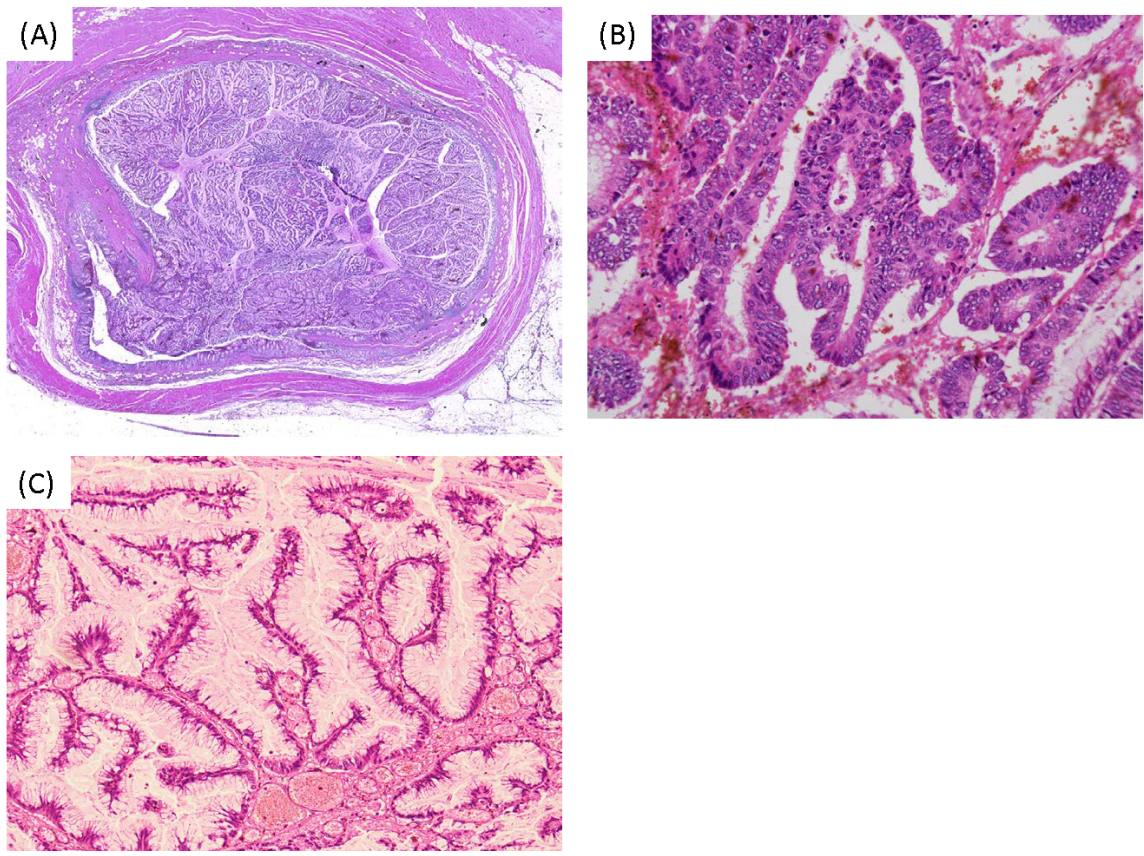